\title{
Planar Near-field Scanner System Error Analysis
}

\author{
Tu Chao ${ }^{1, \text { A }}$, Liu Jun ${ }^{2, B}$, Hu Yinghui ${ }^{3, C}$
}

${ }^{1}$ Vehicle Engineering Department, Changchun Institute of Engineering and Technology, china

${ }^{2}$ Basic Department, Changchun Institute of Engineering and Technology, china

${ }^{3}$ Harbin Institute of Technology, china

afdn_198591@163.com, ${ }^{b} 332552083 @ q q . c o m,{ }^{c}$ foxhyh@163.com

Keywords: planar near field; gantry; error analysis

\begin{abstract}
Planar near-field measurement technology is an emerging technology for near-field antenna measurement. It has been widely used in foreign countries. In recent years, domestic develop faster and faster. Gantry motion platform positioning technology is one of planar near field measurement technology key technologies. It has a direct impact on the measurement accuracy. To achieve high-precision planar near field measurement, the most important is high precision scanning system. This paper analyzes the sources of error and error magnitude of planar near-field scanner system.
\end{abstract}

\section{Gantry design}

Near-field measurement techniques: We use the known properties of the test probe or antenna plane scanning objects within a distance of three to five times the object space operating wavelength. Using fast Fourier transform method for calculating the electromagnetic field of the object scanned the amplitude and phase characteristics, we derive the amplitude and phase characteristics of the object distance field. With its antenna near-field measurements, high accuracy, confidentiality, and small limited range of advantages already in antenna measurement has been widely used.

Gantry plane is an important part planar near field measurement system, which greatly affect the positioning accuracy and scanning flatness of the near-field antenna measurement accuracy. Therefore, the planar near field scan frame error analysis to improve the accuracy of the measurement system is very important.

In this article, we use the inverted T-shaped flat scanner as the research system, to analyze its main sources of error and error magnitude.

The system shown in Figure 1. X-axis made of cast iron or granite. Y-axis is made of rectangular steel tube .We installed two precision ball linear guide in the $\mathrm{X}$-axis surface. $\mathrm{Y}$-axis was moved on the linear guide by motor. We Install a Z-axis stage on the Y-axis surface.Also, Z-axis stage was moved on the linear guide of $\mathrm{Y}$-axis by motor.

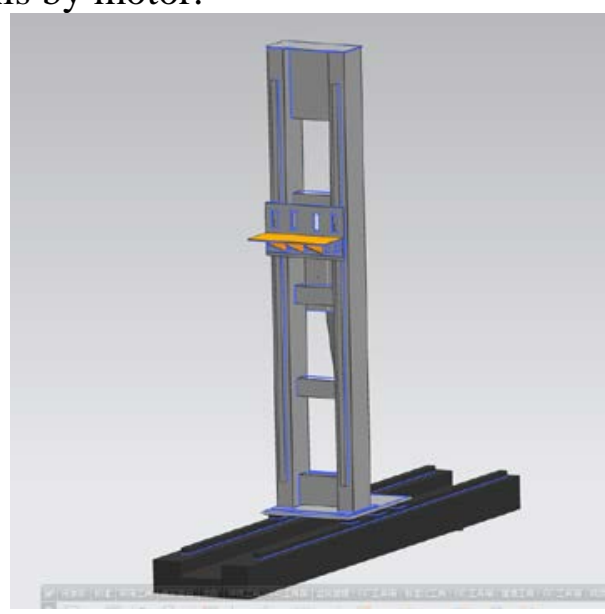

Figure 1 System Diagram 


\section{The analysis of the main source of error}

\section{Assembly Error Generated By The Rail.}

Gantry X, Y-axis guide rail is mounted on the base platform. Due to the impact of manufacturing processes and assembly techniques, will produce installation errors. The screw will have a platform horizontal angle errors during installation.

As shown in Figure 2. $\alpha$ is the axial deflection error angle When installing. This tilt will cause an error occurs in the rail axial length measurements. On the contrary, in the axial tilt also results in a radial length measurement errors, it will also affect the accuracy of coordinate points.

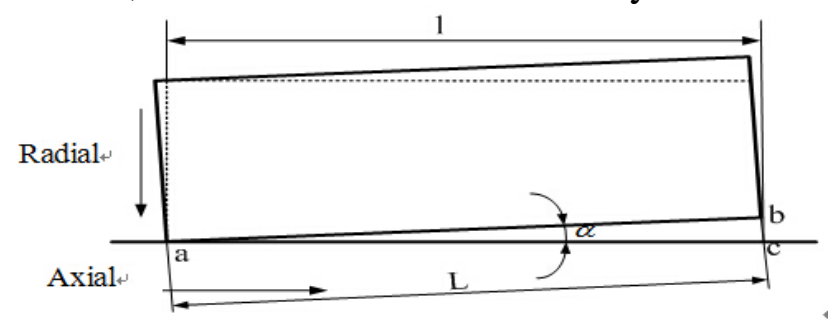

Figure 2 schematic radial tilt guide

Figure 2-1. Setting the distance $L$ is parallel end faces of the guide rail, $\alpha$ is the tilt angle. Therefore, there are different between the measuring line ac and the measuring line ab, the actual measured value l, the true value of L, both satisfying the formula (1), the measurement error satisfies the equation (2).

$$
\begin{gathered}
L=l \cos \alpha \\
\delta l=l-L=l-l \cos \alpha
\end{gathered}
$$

We made measure errors Taylor series expansion, and omit more than three times higher, so we got (3)

$$
\delta l=\frac{1}{2} l \alpha^{2}
$$

Axial error's uncertainty and radial error's uncertainty is not relevant. Length standard uncertainty caused by the installation of formula (4) below.

$$
u_{l}=\sqrt{\left(\frac{1}{2} l_{\mathrm{X}} \alpha_{\mathrm{X}}^{2}\right)^{2}+\left(\frac{1}{2} l_{\mathrm{Y}} \alpha_{\mathrm{Y}}^{2}\right)^{2}}
$$

Formula (4), ul- length standard uncertainty caused by mechanical, IX-X-axis rail length, $\mathrm{IY}$-Y-axis rail length, $\alpha \mathrm{X}$ - axially mounted angle, $\alpha \mathrm{Y}$ - radial mounting angle.

Thermal Error.

The main source for the entire system is a precision ball screw drive system. Its main source of heat is the heat that bearing swivel caused and generated betweenin the the internal ball and screw friction in the process of moving. The factors that affect the heat generated are nut traveling speed, continuous working time, and the use of cooling lubricants and other properties.

\section{1) Heat generated by the ball screw nut}

The formula is:

$$
Q_{1}=0.12 \pi n M
$$

$n$ : Ball screw rotational speed, the unit is $(r / \mathrm{min})$

$M$ : Nuts friction torque, the unit is $(N . m)$

Check the user manual, $M=9.8 \times 10^{-2} N . m, n=100 \mathrm{r} / \mathrm{min}$ (normal use, the use of non-fast).

$Q_{1}=0.12 \pi \times 100 \times 9.8 \times 10^{-2}=3.7 \mathrm{KJ} / \mathrm{h}=1.03 \mathrm{~J} / \mathrm{s}$ 。

\section{2) Heat generated by the Ball Bearings}

The formula is:

$$
Q_{2}=1.047 \times 10^{-4} \times n M
$$




$$
\begin{aligned}
& v n>2000 C S t \cdot r / \min , M_{0}=10^{-7} f_{0}(v n)^{\frac{2}{3}} d_{m}^{3} \quad(\mathrm{~N} . \mathrm{mm}) \\
& v n<2000 \mathrm{CSt} \cdot r / \mathrm{min}, M_{0}=160 \times 10^{-7} f_{0} d_{m}^{3} \quad(\mathrm{~N} . \mathrm{mm}) \\
& d_{m}: \text { Bearing diameter in the middle }(\mathrm{mm}) ;
\end{aligned}
$$

Find available from the user manual, screw starting torque $0.32 \mathrm{~N} . \mathrm{m}$, ball screw speed of $100 \mathrm{r} / \mathrm{min}$ to calculate the bearing calorific value: $0.6 \mathrm{~J} / \mathrm{s}$

Through simulation, the thermal effect analysis diagram can be drawn, heat transfer per unit time is not obvious, both at $25^{\circ}$ or less. The heat deformation maximum offset of $0.0025 \mathrm{~mm}$.

\section{Load Error.}

Part of the weight of the platform is pressed against the ball screw. Gravity makes the ball screw in the Y direction by a downward pressure. So that the screw in the force generated at the angle and offset errors. This error will lead to the Y-axis and the probe movement along the dotted line. Ultimately makes the probe position appears positional deviation in the $\mathrm{X}$-axis and $\mathrm{Y}$-axis.

Fixation screw is fixed at both ends, it can be reduced to material mechanics fixed at both ends, in the middle by the concentrated load beam model. Diagram below, in which:

$\mathrm{P}$ is the removal of the weight acting on the two outer rails, acting on the ball screw load force, the unit is $\mathrm{N}$.

$x$ is the working platform is the distance from the side of the nut.

$l$ is the effective length of the entire ball screw.

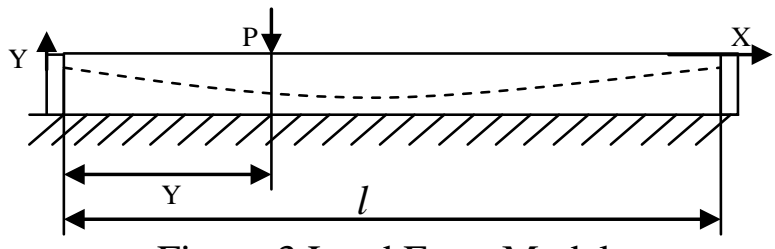

Figure 3 Load Error Model

According to the formula, we can see

(1)Angular error

$$
\theta=-\frac{P(l-x)\left(l x-2 x^{2}\right)}{3 l E I}
$$

$E$ : Ball elastic modulus.

$I$ : Screw-sectional moment of inertia of the neutral axis.

$E I$ : Bending stiffness screw material.

(2) Offset error in the Y-axis:

$$
\Delta Y=-\frac{P(l-x)^{2} x^{2}}{3 l E I}
$$

Sign on behalf of the screw in the offset direction.

\section{Conclusion}

This paper analyzes the main error sources of gantry in addition to the original rail screw error.We can be drawn from the analysis, the middleweight of thermal error is much smaller compared to other error. Therefore, it can not be considered in the design and compensation. The assembly errors are a major source of systematic error.

Therefore, it should be possible to improve processing accuracy, reduce assembly errors in the manufacturing process and assembly. We should mainly compensate assembly error when error compensation. 


\section{References}

[1] Wang shukun. Ball Screw Feeding System Positioning Accuracy Analysis [J]. Dalian University of Technology (Master's Thesis),2006 (12).

[2] Ding haifeng. Study XY table positioning error of dynamic measurement and compensation system [J]. Shandong University (Master's Thesis),2008 (05).

[3] Lei ting. Automatically detects the thickness of the non-contact development system [J]. University of Electronic Science and Technology (Master's Thesis),2013 (06).

[4] Zhang fushun. Error Analysis and Compensation ultralow sidelobe antenna planar near field measurement [J]. Xi'an Electronic Science and Technology (doctoral thesis),1999 (12). 